\title{
Negotiating Intelligence, Nerdiness, and Status in Physics Master's studies
}

\author{
Anders Johansson ${ }^{1,2}$ \\ Published online: 14 November 2018 \\ (C) The Author(s) 2018
}

\begin{abstract}
Motivated by widespread concerns for representation and equity in physics education, this study investigates negotiations of identity positions of master's students in physics. The goal is to explore how sociocultural features of physics can structure the possibilities for becoming a physicist. Interviews with international master's students in physics were analyzed focusing on perceived norms about physics and how students responded to and negotiated these in crafting a position as competent physicists-to-be. The results show how physics master's students from varying backgrounds have to negotiate stereotypes of intelligence and nerdiness, as well as an implicit ranking of physics specializations. The stereotype that physicists are intelligent and nerdy is further complicated in physics studies, as some specializations - the more pure and theoretical - are viewed as more intellectually demanding and are in this way accorded a higher status. Students on these specializations are simultaneously seen as more intelligent and more nerdy, while a perception that students who choose other subjects do this just because they are not good enough to do theoretical physics is perpetuated. These notions become significant in relation to western society's high regard of authentic intelligence and idolization of geniuses, ideas that can serve as powerful ideals in physics. This study gives novel insight about how well-known norms and stereotypes about physics come to matter in physics master's students' negotiations to become recognized as competent physicists.
\end{abstract}

Keywords Social identity · Physics education · Graduate education · Equity · Discourse

\section{Introduction}

Physics is commonly regarded as a prestigious subject, and comparatively, few university students complete a degree in physics. Concerns about the underrepresentation of women and minorities in this group have motivated researchers and practitioners to try and solve the

Anders Johansson

anders.johansson@physics.uu.se

1 Department of Physics and Astronomy, Uppsala University, Box 516, 75120 Uppsala, Sweden

2 Centre for Gender Research, Uppsala University, Box 527, 75120 Uppsala, Sweden 
gender gap of physics, for example via efforts for making women more interested in physics (Macdonald 2014). However, a growing body of research that takes its departure in theories about social identity has turned the problem around to focus on the construction of norms in physics, rather than seeing the underrepresented groups themselves as a problem (Archer et al. 2016; Johansson 2016; Traxler et al. 2016). The goal for this kind of research is to analyze students' interactions with the subject culture, how they relate to norms and construct identities. When it comes to physics, research has shown how it is not only often conceived of as inherently masculine, difficult, and requiring a brilliant intellect but also at times as a subject for nerds. These notions have been shown to restrict the possibilities for being recognized as a physicist (Archer et al. 2016; Francis et al. 2017b; Gonsalves et al. 2016; Gonsalves and Seiler 2012).

This article adds to the picture of social identities in physics by analyzing interviews with a previously little studied group, international first year master's students in physics, and focusing on how they have experienced their path to becoming physicists. While research about identity and inclusion in physics has often focused on newcomers or research environments, students on the master's level are well on their way into the physics discipline, although they cannot be perceived as complete insiders. This means that they can provide valuable insights about how more or less well-known norms about physicists are negotiated in physics departments throughout the world. The notions of physics as a subject for intelligent and perhaps nerdy people are well-documented in the literature, and an implicit ranking of physics subjects may be familiar to any physicist, but how these norms come to matter for experienced physics students in navigating their studies has not been investigated in-depth in the research literature before. This study thus represents a first endeavor for examining physics master's students' negotiations in relation to these norms. Building on previous work on identity in science education, I will take my departure in poststructuralist accounts of identity and gender, in particular, the notion of identity as a question of negotiation of positions given in discourse.

With this background in mind, the aim of this article is to explore the negotiations of positions in physics master's students' trajectories towards becoming physicists in relation to perceived norms about physics and physicists. In particular, how do discourses about intelligence, nerdiness, and a relatively higher prestige of pure and theoretical physics come into play?

\section{Background-Social Norms and Identity in Physics}

Previous research about the culture of physics and social identity has identified several characteristics that may be barriers to an inclusive physics culture. In one of the now classic studies of the field, Sharon Traweek showed how the particle physicists she studied seemed to regard physics as a culture of no culture, appealing to the supposedly value-neutral nature of physics knowledge. She could nevertheless describe how multiple cultural beliefs about physicists structured the environment and for example worked to exclude women (Traweek 1988). In recent times, physics has been found to be perceived as a masculine and difficult subject by students in school and their parents (Francis et al. 2017b). These findings correspond to discussions in feminist studies of science which suggest that science in general holds masculine or androcentric values (Harding 1986; Keller 1985; Merchant 1989). 


\section{Research About Social Identity in Physics}

In research about social identity in physics, many researchers have focused on the notion of recognition, asking what is needed to be recognized as a legitimate and competent physics student (Carlone and Johnson 2007). Notions of recognition and competence have been used as factors in statistical modeling of physics identity (Hazari et al. 2010, 2013), but the research closest to the interests in this article draws from a qualitative research tradition inspired by poststructuralist accounts of power and discourse. In this research, being recognized as a physicist means performing an intelligible identity by negotiating subject positions given in discourse. Allison Gonsalves (2014) has identified how certain ways of performing an identity as a competent physicist are more easily recognizable and differentially available to women and men. Gonsalves and Seiler (2012) argue that, even though the stereotypical picture of the physicist may seem to exclude women, a number of ways of displaying competence that are not always included in the stereotype may provide additional paths to achieving recognition. Several studies have nevertheless pointed out how a position as a physicist generally excludes femininity, particularly performances of what can be termed stereotypical or girly femininity (Danielsson 2009; Gonsalves 2014). Getting recognition among physics students may also be related to performances of playfulness or demonstrating a knowledge of common cultural expressions, like science fiction, something which again carries gendered connotations (Hasse 2008, 2015).

Different fields of science have proved more or less available to women and other minoritized students (Leslie et al. 2015). This also holds true within physics, where the areas considered harder, both in terms of being more difficult and more scientific, are statistically more dominated by men (Hasse 2015; Hasse et al. 2008; Hasse and Sinding 2012). However, there are many different possibilities for navigating these norms about identity, competence, and suitable physics interest. For example, high school girls who identify with physics have been found to prefer to align with theoretical physics (Archer et al. 2016). In this way, they emphasize their analytical competence, and perhaps avoid the more strongly masculine connotations of experimental/technical ways of doing physics.

\section{Curious, Nerdy, Intelligent-Characteristics and Stereotypes About Physicists}

One aspect of the negotiation of discourses in physics concerns the expected characteristics, or stereotypes, about physicists. Identifying with physics may carry a connotation of being nerdy or geeky, but usually at the same time clever and absorbed in one's subject, corresponding to the image of the stereotypic scientist (DeWitt et al. 2013).

A significant driving force for physics students seems to be a genuine and deep interest and fascination with physics, or more generally for understanding the universe (Bøe and Henriksen 2013; Levrini et al. 2017). This fascination has been reported as something awakened already in childhood for many physicists who describe that they have "always" wanted to do science (Maltese and Tai 2010). However, the narrative of the child physicist is also a way of performing an authentic identity as a physicist, putting beyond doubt the legitimacy of one's place in physics (Hasse 2008). This display of passionate interest may also incidentally or intentionally be part of aligning with a "nerd" identity (Willey and Subramaniam 2017).

The figure of the nerd, or geek, is commonly imagined as a White male, lacking social skills, and unsuccessful in the heterosexual market, with some sort of technical/scientific competence and often engaged in some kind of nerd subculture (Eglash 2002; Kendall 
2000; Wajcman 1991). In school, being good at science and deemed a nerd, or, in British usage, a boffin, often means being the subject of derision (Francis 2009). Nevertheless, the title can be appropriated as a badge of honor in communities where the scientific capital associated with it is highly valued (e.g., in groups of university students, see Mendick and Francis 2012). Nerdiness can in this context be viewed as an attitude that is useful and more or less expected of a certain type of scientist. This is how the term is sometimes used by the physics graduate students interviewed by Danielsson (2009) and Gonsalves (2014). Identifying as a nerd may also be a way for women to perform a science inclined identity, which may however be perceived as conflicting with femininity (Archer et al. 2016). In a sense, the general view of the nerd can be understood as one of reverent disdain, where the asocial, failed masculinity of the nerd is disdained at the same time as the scientific credentials of the nerd are revered, in line with the high regard for science in society (Willey and Subramaniam 2017).

With the image of the nerdy physicist also follows the notion of cleverness or intelligence. The most classic picture of a physicist is also our culture's most classic picture of a brainy genius, captured in the figure of Albert Einstein with his hair on end. Although the nerd may be disdained or a figure of abjection, western culture has a love affair with authentic intelligence (Castles 2012; Dweck 2006). The idea of intelligence as a more or less stable trait of an individual traces its roots to nineteenth century science, and the eugenics and psychometric movements developed by Francis Galton and others, but can be recognized also today for example in notions of gifted education or ability grouping (Francis et al. 2017a; Privateer 2006). One prominent issue with the idea of authentic intelligence, or of the genius, is the gendered, racialized, and classed connotations. For example, intelligence testing has from the beginning been associated with dubious claims about the natural superiority of already privileged groups (Privateer 2006), and this association also plagues discussions about giftedness to this day (Kerr and Kronborg 2015). Discourses that position boys as more authentically intelligent and girls as more hardworking are widespread inside and outside education and seem hard to do away with (Paule 2015). While hard work, or collaboration, can be seen as valuable behaviors, the raw intelligence of the genius still evokes more respect (Paule 2015), and a position as an effortless achiever is thus desirable in many education contexts, but often more readily available to white middle-class boys/men (Jackson and Nyström 2015). The gendering and racialization of notions of intelligence and brilliance seems to be one mechanism whereby women and racialized academics are discouraged from pursuing certain academic fields such as physics where the beliefs about the necessity of raw talent are stronger (Leslie et al. 2015; Storage et al. 2016).

In conclusion, earlier research has pointed to how physics is generally considered a difficult subject, suitable only for deeply interested and intelligent people, who may by virtue of this be considered nerdy. Importantly, these stereotypes have been found to be potentially limiting to the participation of women and minorities in physics. This article details how these common discourses around physics and physicists come to matter for master's students in physics, a previously understudied group.

\section{Positions Negotiated in Discourse-Theory and Terminology}

Research about social norms, identities, and gender in physics has used a variety of theoretical frameworks and methodological approaches. Most studies discussed in the previous sections draw from a social constructionist perspective, where identity is seen as co-constructed in the interaction of social actors and generally not as a characteristic possessed and retained by 
individuals (Pozzer and Jackson 2015). In this article, I follow this tradition and use a poststructuralist account of social construction, where identities are viewed as constituted in discourse. Discourses, in my use of the term, denote entire systems of meaning-making, the frames of language, and other systems of signification through which we understand the world (Laclau and Mouffe 1985). There is then no way of going outside discourse in this picture. However, a discourse can never be total; dominating discourses can always be resisted or changed through constructions of opposing discourses or re-articulations of terms (Laclau and Mouffe 1985). This can be described in terms of a struggle where some discourses achieve hegemony, a temporary dominance in defining how we understand the world (Laclau and Mouffe 1985; see also Wetherell and Edley 1999).

Personal and social identity in this poststructuralist framework is conceptualized in terms of subject positions. Identities are "points of temporary attachment to the subject positions which discursive practices construct for us" (Hall 1996, p. 6). This does not however imply a totally determinist view where people are only products of social structure; rather resistance and change are possible. This is captured for example by Judith Butler's (1990) account of the institution of identities through repeated performative acts, which refer to already established discourses, but which may also offer resistance and change through failed or parodic repetitions. Butler describes how a prevailing discourse establishes some positions as intelligible and some as unintelligible. In elaborating these concepts in science education, others have discussed this in terms of recognized (capturing both intelligibility and appreciation) and celebrated (meaning the most valued) identity performances (Archer et al. 2017; Carlone et al. 2014; Carlone and Johnson 2007; Gonsalves 2014).

In the analysis of this article, the focus lies on how the interviewed students make sense of themselves. That is, how they relate their own position to subject positions made intelligible in discourse. One aspect of this concerns relating to established hegemonic, ideal, or celebrated subject positions. This is done both in active negotiations and in implicit affirmations of the rules of a current discourse. As a way of attending to this process in practice, I borrow the notion of negotiation of imaginary positions from Wetherell and Edley (1999), who use this to discuss how men relate to hegemonic masculinity (Connell 1995). In this framework, negotiations around positions given in discourse can involve an investment in a given position, complicity in accepting it but not inhabiting it, or resistance in rejecting it (Wetherell and Edley 1999). In the specific context of physics, I use this notion to capture how the students make sense of themselves as physics people in relation to "imaginary positions" given as norms or stereotypes about who one should be as a physicist.

In doing this, I am not primarily focusing on identity categories, such as gender or ethnicity, but rather on the conditions for identification in physics education. However, all subject positions carry gendered (etc.) aspects, and as we have seen in previous research, this is very much the case for the stereotypic expectations of physicists as well.

In drawing upon these theoretical and empirical backgrounds, a few central notions were used for making sense of the interviewees' accounts: intelligence, nerdiness, and authenticity. A few comments about these concepts are in order. Intelligence as well as nerdiness are mostly drawn from the data, where my interviewees make explicit references to being smart, a genius, or a nerd. However, I also interpret statements such as people being considered crazy for choosing a very difficult subject such as physics to be part of the nerd complex of ideas which, as pointed out above, includes passionate or obsessive interest for specific topics. In this article, nerdiness generally refers to these characteristics and not to consumption of popular culture from "nerd" genres, such as sci-fi or comics. Authenticity is often expected in presentations of the self. You are 
expected to be true to your identity, or to possess the right qualities to pass as a certain kind of person, that is, not being a fake. However, a poststructuralist account of the performative construction of identities challenges this view by pointing out how authenticity is rather constructed and reconstructed through personal narratives of continuity, claims of "always" having been a certain way (Mendick et al. 2009). It has been demonstrated that such narratives are often quite unconsciously changed and adapted to the situation, for example in descriptions of interest and study choice (Holmegaard et al. 2015).

\section{Materials and Methods}

The material used for this study is drawn from a project investigating discourse and identity on physics master's programs. The project was conducted using participant observation and semistructured interviews, but this particular article focuses on the narratives given in interviews with ten students, while the observations, along with my own experiences as a physics student, are used as a background to understand the context of the master's programs. The students had all studied a 3- or 4-year bachelor's program to qualify for the master's program they were now taking at one of two large Scandinavian research-intensive universities. In Scandinavia, master's programs are usually offered with a few specific specializations that students can apply for. At the universities in this study, the students in each specialization usually attended the same classes, particularly during the first semester, during which the students were interviewed. A list of the students and the specializations of their master's program are given in Table 1.

An understanding of the distinctions within physics as it relates to the choices of specialization for the interviewees is central to the analysis. In general, physics subjects can be placed along the dimensions theoretical — experimental and pure - applied. While theoretical physics has traditionally been more pure, i.e., basic research, experimental physics can be oriented towards both applications and basic general understanding. Traditional divisions among these fields generally accord higher status to the theoretical, pure, and supposedly more fundamental physics (Becher and Trowler 2001; Traweek 1988; Whitten 1996). The specializations of the interviewed students are directed towards either theoretical or experimental physics, although most subjects have both theoretical and experimental parts. For example, the theoretical physics specialization explicitly excludes experiments, while meteorology and geophysics

Table 1 The interviewed students, the specialization of their master's studies, and whether this specialization is considered theoretical, experimental, and pure or applied

\begin{tabular}{lll}
\hline Interviewee & Master's specialization & Direction of specialization \\
\hline Alex & Materials physics & Experimental, often applied \\
Bo & Materials theory & Theoretical, often applied \\
Dan & Geophysics & Experimental/theoretical, pure and applied \\
Elias & Medical physics & Experimental and applied \\
Eugenia & General experimental physics & Experimental \\
Ivo & Theoretical physics & Theoretical, primarily pure \\
Jan & Theoretical physics & Theoretical, primarily pure \\
Sara & Nuclear physics & Experimental, often applied \\
Susanna & Space physics & Experimental, often applied \\
Valter & Meteorology & Experimental/theoretical, pure and applied \\
\hline
\end{tabular}


can be both theoretical and experimental. Space physics and materials physics are conceived of as more applied fields, since the experiments can often be expected to have practical or commercial applications in the near future.

All of the interviewed students had moved from the country where they had grown up and received their bachelor's degrees, and all interviews were conducted in English, which was not the first language for either the interviewer or the interviewees. ${ }^{1}$ The ethical guidelines set out by the Swedish Research Council and relevant bodies in the countries of the study have been observed throughout the project. Before observation and interviews took place, the general goals and procedures of the project were discussed with students, and the interviewed students agreed to participate and have the interviews recorded on the basis of a detailed description of research goals and procedures.

The interviews lasted between 50 and 100 min each and focused on three broad themes: Experiences of studying physics, experiences of starting on the master's program, and ideas about what it means to be a physicist. A few examples of the exploratory questions used are as follows: When and why did you decide to study physics? What were your expectations for this master's program? Were you interested in a specific kind of physics when you started university? Have you changed your views? Why? Would you call yourself a physicist? What are the characteristics of a typical physics student? A good physics student?

The analysis draws from a qualitative, interpretative research tradition, which means that the findings are the results of the interpretative work done by the researcher (Taylor 2014). Transcription of the interviews was done roughly at an initial state and a preliminary thematic analysis was conducted. From this, a preliminary research question focusing on negotiations of positions in relation to physics specialization was formulated. After this, detailed verbatim transcription was done, excluding a few parts of the interviews deemed less relevant for this question. An open coding process was used to identify constructions and contestations of what a competent physicist is and how students negotiated their place in physics in relation to this. For example, the code "physicist" linked all statements of how a physicist is, should be, or is perceived, with sub codes like "physicists vs engineers," "physicists are viewed as smart," "physicists are viewed as weird," and "physicists are altruistic" pointing to various aspects that could be recognized in several discussions. Using these codes, thematic summaries were written to answer specific questions asked to the material such as: "In what ways do the interviewees compare different kinds of physics and how do they position themselves in relation to them?" or "How have the interviewees experienced the perceptions of others about physics students?" During the interpretation work, interview transcripts were re-read and the coherence (or lack of coherence) of each interviewee's narratives was considered. The results were interpreted in the light of the poststructuralist notions of negotiations of imaginary positions within discourse described above. In analyzing the material in this way, intelligence, nerdiness, and status stood out as a central and general topic for most of the interviewed students. The analysis of this article therefore focuses on this theme, while a few other themes found in the analysis are not included in the scope of this article. One theme concerned specific stories about the undergraduate quantum mechanics course, which a few of the interviewees had experienced as particularly important for their path through physics. This theme has been

\footnotetext{
${ }^{1}$ For confidentiality reasons and the risk of in-group identification, I refrain from reporting the nationality of the individual students. In this article, their specialization in physics was deemed more important. The national background of the students were Belgian (one), Chinese (one), German (one), Greek (two), Italian, (two), Mexican (one), Polish (one), and Spanish (one).
} 
explored and reported elsewhere (Johansson 2018). Another theme that is not included in the current analysis concerns how some of the interviewees compared their previous university to where they were studying at the time of the interview. A handful of statements suggested that the current (relatively prestigious) Scandinavian universities were better than a home university in southern Europe, at least in the sense of having prepared the local students better for the master's program. A part of this may perhaps be attributed to a kind of confirmation bias: having just made an active choice and perceiving it as better than other possible choices. Even though this theme indicates interesting points in relation to the status of physics studies, the material was not conclusive enough to explore the topic further.

\section{Being a Physics Person in the Eyes of Non-physicists}

Subject positions are always defined in terms of differences to others. The findings presented here will deal with the question of positions as physics people in two stages. This first findings section will describe how the interviewees have experienced a position as a physics person to differ from other common positions for young people. In particular, this points to how associations of intelligence or nerdiness with physics may be a social stumbling block, both for those who fit easily into the stereotype and for those who do not. The imaginary position of the weird, nerdy or intelligent physicist intersects with gender and social background in the negotiations of the interviewees. These findings resonate with earlier results and serve as a background to the differentiations within physics that are elaborated in the next section.

Many of the interviewees described how they have been seen as not only smart but also as nerdy or otherwise different in high school and in interactions with people outside physics. All of them were interested and good at science in high school, and while this could lead to encouraging praise by parents and teachers, it could also mean being the butt of jokes in a school culture where educational success is suspect. This happened to Jan, who described how his friends in school were always "kidding about" him, often in reference to him being a "future Sheldon" (referring to the archetypical nerdy physicist Sheldon Cooper from the popular TV-show The Big Bang Theory). In Jan's experience, these jokes were friendly enough, and did not mean he was cast out in school. The interviewees recognized how people in general usually associate doing science with intelligence and nerdiness. Bo described that if he presented himself as a physics student, "some people will think 'oh you are crazy," and he explained how this craziness is associated with doing intellectually difficult things, being "some kind of genius." This problem of presenting yourself as a physics student was described by several of the interviewees. These accounts of the views of physics as nerdy and smart and of nerdy school pupils are in general in line with earlier research (Archer et al. 2016; Francis et al. 2017b; Mendick and Francis 2012).

While a few of the interviewees acknowledge the idea that you actually have to be smart to do physics, this notion was challenged by Jan. Jan, like others, described that when he presented himself as studying physics, people's reaction would be to frame the subject as: "Oh it's a hard one. Wow, it's very intelligent." He said that he then usually tried to explain that he was no genius and that most people actually could study physics, but that everyone seems to think it is too hard. That Jan resists this common stereotype may be interpreted as him recognizing that being considered some kind of genius would be too big shoes for him to fill.

In earlier research, it has been pointed out how discourses about intelligence and nerdiness in physics or science often carry gendered connotations, with the stereotypical image of a 
genius or nerd being male (Archer et al. 2016; DeWitt et al. 2013; Francis 2009). Several of the interviewees described how these gendered preconceptions about physicists affected them and the expectations people put on them. One example of this is discourses around being more or less attractive as a nerdy guy, recognized by several of the interviewees. When discussing the issue of presenting yourself as a physics student Bo and Valter touched upon this point, that you may be seen as too nerdy "among the girls." This is evident in Valter's description of the problem of introducing yourself:

And I think that the average people think that you are smart because everybody has this comparison with Einstein and everybody thinks that Einstein was the best person who ever lived on the earth, and he was a physicist [...] Also sometimes, it's not the best way to ... eh introduce yourself with the girls because: 'I study physics' [in a quiet voice:] 'oh ... nice'

I interpret this statement as indicating that being a male physics student is associated with nerdiness, making one less desirable on the heterosexual market but still perhaps retaining some of the status implied by being viewed as intelligent or competent. This doubleness of the male nerd position has been noted in earlier research (Kendall 2000; Willey and Subramaniam 2017).

In Valter's discussion of his position as a physics person, he also in some sense constructs different positions for women in physics and outside physics. He continued his discussion of the low desirability of men in physics by explaining that "probably it's because of that that my girlfriend is a physicist." Although this statement may present the girlfriend as smart and able to see beyond stereotypes, it also in some sense sets her apart as different to most women. A female physicist is not simply one of "the girls." Susanna spoke at length about the stereotypes about physicists and noted how people often attempted to match her to those stereotypes:

For example being a physicist woman is something very weird for people who are not into this field like 'oh you are a physicist and you are a girl. Oh you are so weird'. Because they don't have a stereotype of a (laugh) girl being a physicist. If you are like, a guy, ok you are into this in a nerdy stuff and you spend all your time alone and you don't have a girlfriend. But if you are a girl, they cannot, like, put you in a stereotype box (laugh).

Susanna here points to how the intelligible imaginary position of the physicist is so strongly associated with masculinity, at least to the outsiders she refers to here, that being a woman physics student can seem completely unintelligible. This corresponds to a double association of both physics and nerdiness with masculinity. It has been demonstrated earlier that under these expectations it can be difficult to combine a position as a competent physicist with feminine gender performances (Archer et al. 2016; Gonsalves et al. 2016; Madsen et al. 2015; Ong 2005).

The themes of difference, intelligence and nerdiness also intersect with the social background of the students. Being seen as different in some way because of one's interest in physics was a stronger theme for those of the interviewees who had grown up in a small town, or came from a working-class background. This difference could however be a positive experience. For example, both Sara and Valter described how they were one of few students who were good at science in their school and how their surroundings were proud and encouraging. Sara said that everyone expected her to go on with physics, as she was "a really nerdy pupil." I interpret this to mean that an "exceptional physics girl" (Archer et al. 2016) can get recognition and encouragement, and that nerdiness can be seen as a positive characteristic 
in the right context, in this case the small town where Sara went to school. Sara later reflected back on how her path put her at a distance to her old friends, who may have stayed in the home town and had already married and had children. Choosing physics for Sara thus meant some distancing from traditional (small town or working class) femininity.

The experience of being one of few science inclined students in a small town school was not described as positive for all of the interviewees. For Susanna, going to university and leaving her small home town was experienced as a liberating move, especially since the expectations for women in her home town did not fit with her interests. She said that in her home town "nobody knew what physics is," while engineering, was recognized as a "good job." In particular, "if you are a girl and you study physics, it's something that doesn't belong to this world (laugh)." Choosing to move away from this small town context was then not only necessary for being able to study at the university, but moving also became a way to find an environment where she could fit in better. In Susanna's words: "My hometown is small so everybody fits in a box, and I was out of every box they had. And I felt that I had to change because it was not a good environment for me." Here, we can see how becoming recognized as a physics person does not only concern having the value of that position acknowledged, but that it can also involve finding a context where this position is intelligible at all.

Susanna's story is one example of the many negotiations around positions as physics people in the interview material, and it points to some of the significant issues of becoming a physicist. Physics is a subject associated with intelligence and nerdiness, and if you are a woman growing up in a small town, it is difficult to align to the imaginary position of a nerdy genius. Even though being positioned as a nerd is often an abject position (Francis 2009), a position as intelligent or nerdy may also be positive. This can be the case in the right school, but especially at the university, where one often meets like-minded people (Mendick and Francis 2012). The accounts of the interviewees point to how the figure of the physics nerd is associated with intelligence or competence that can be "revered" at the same time as the perceived asociality of the nerd is "disdained" (Willey and Subramaniam 2017). Most of these findings corroborate earlier results concerning the discourses about physics encountered by students.

\section{Negotiating Positions within Physics}

One part of becoming a physicist concerns being recognized by others as such and negotiating a position that sets oneself off as a physics person. How the interviewees experienced this differentiation to others was described in the previous section. However, another part of the journey concerns being recognized as a competent, legitimate participant in the community of physics students and physicists. Here, another level of differentiation becomes important, relating to which kind of physicist one is to become. In the accounts of the interviewees, this struggle for recognition had to be done in relation to discourses that posit some physics subjects as better than others, mostly in terms of being more difficult and demanding more intelligence. These evaluations tended towards celebrating pure and theoretical physics more than applied and experimental. The negotiations of physics positions in this context meant that a choice of the less celebrated subjects in some cases necessitated a need for a defense against being viewed as not smart enough. In the discourses of physics studies, interest, passion, intelligence, and nerdiness were some of the characteristics of the celebrated imaginary physicist positions the master's students had to negotiate. While these notions are well- 
known when it comes to physics, the results presented here give a detailed account of how they can come to matter for students in the middle of their physics education.

\section{Authentic, Passionate Interest in Physics}

Being recognized as a good physics student can mean not only displaying the right competence or intelligence but also showing the right kind of attachment to physics. In general, research shows that the decision to study physics is more often driven by an interest for the subject rather than a desire for getting a good job (Bøe and Henriksen 2013; Levrini et al. 2017). Several of the interviewees' describe how they were more encouraged to go into engineering by parents or others, but how their choice of physics defied some of these expectations. Following one's interest in physics was then experienced as liberating. However, the discourse of physicists as interest-driven passionate nerds was experienced as particularly restrictive by one of the students. For Susanna, not conforming to the idea that interest in physics should be driven by a deep-seated curiosity was something that she experienced set her apart from the typical physics student. Susanna said: "No, I'm not like a common physics student but only because physics was not my dream. I think that a typical physics student is someone who dreamed about physics for his whole life and for me, it's not like that." Susanna explained that she had been wanting to become a hairdresser in her late teens, and that she was still uncertain about her goals and future career at the time of the interview.

While some kind of curiosity seemed to be a driving force for most of the interviewees, the authentic life-long interest that Susanna refers to as common among physics students was not typical among the interviewees. On the one hand, Jan describes how he started to think that physics or some other kind of science would be a way of getting a deeper understanding of the universe, when he got inspired by reading the fantasy book-series His Dark Materials (Pullman 1995-2000) as a young teen. On the other hand, Eugenia claims that she liked mathematics but hated physics and learned to like it only when she got a good high school teacher who could help her to better see how it all fitted together. Still, Eugenia did not consider physics a viable career until after having started her university studies in architecture, from which she subsequently switched to physics. In general, all the interviewees report that they were in some way good at science in school (some, like Eugenia and Sara, even won math competitions) and were encouraged to carry on along that general path by parents and/or teachers. However, physics was not described as an obvious choice by any of the interviewees. Judging from this small group, dreaming about physics your whole life does not seem to be the norm for physics students. Rather, insecurity about one's study choice and a late-awakened interest are also common.

I interpret Susanna's hesitation about her place in physics as being predicated upon her position as a female physics student who grew up in a non-academic context. For her, physics is not an obvious choice, but she perceived it to be more so for others. The story of the "child physicist" (Hasse 2008) serves as an imaginary position for an authentic physicist, who has "always" been that way (Holmegaard et al. 2015; Mendick et al. 2009). At the same time this construction means that students, like Susanna, who do not fit this description are viewed and view themselves as less authentic physics people. Being recognized as a proper physics student may at times involve being seen as a "passionate nerd" and showing a deep attachment to physics as something you have always been aiming for. This was however not the case for the majority of the interviewees. 


\section{Implicit Rankings of Subjects-Physics Is Better, Pure Theoretical Physics Is Best}

In discussing their educational choices, the interviewees drew from discourses ascribing different values to different academic subjects. In general, and perhaps unsurprisingly, since it is what they have chosen, physics was often viewed as "better" than other subjects in some way. However, not only was physics viewed as better. In the stories of the interviewees more theoretical and pure physics also had a higher status than experimental and applied physics. This evaluation mirrors the general characterization of the "most favored specialisms" in academic subjects (Becher and Trowler 2001, p. 192), but it also reflects ideas about what demands more or less intelligence.

Some explicit comparisons between physics and other subjects were given by students who had switched to physics after having started their university studies. For example, Eugenia initially studied architecture, but after the first years found that she wanted more intellectual challenges:

[Architecture is] being a craftsman, not somebody that thinks. It's more, you're the hands not the brain. So after one year of drawing and not using my brain I was like 'I hate it (laugh), I want to do physics just because I like it' and I signed up for physics at like, eh, the last possible moment.

Another difference that Eugenia noted is that intelligence is what counts in physics; people "respect you more if you are smarter." This is in contrast to architecture where clothing style and good taste were perceived as important status markers. Bo compared materials engineering, which was his first subject at the university, to physics, which he switched to after being inspired by taking quantum mechanics. He said that he would be more proud of having a physics degree and that physics is "cool" compared to materials engineering. He explicitly, but also jokingly and perhaps influenced by his negative experiences on the earlier program, evaluated subjects along an axis of coolness from mathematics to physics over chemistry to engineering, positing materials engineering in particular as "not cool" and "boring." In Bo's view, materials researchers mostly "just do experiments repeatedly" and "just change the parameters again and again."

Although physics, as has been discussed above, was associated with intelligence, not all kinds of physics connote the same picture of a brainy genius. Several of the interviewees spoke about the general association of physics with figures like Albert Einstein, or perhaps Sheldon Cooper. These figures are theoretical physicists, with a strong theoretical intellect, but are also often imagined as introverted, or even eremitically reclusive, asocial, asexual, male geniuses. This picture can perhaps agree with some ways of doing physics, but to a large extent excludes the collaborative and practical nature of most of experimental (and often also theoretical) physics. Alex, who studies materials physics, noted that people in general "have the idea that a physicist is a theoretician," which means his ideals of doing physics in an experimental and collaborative way, are not immediately recognized. Sara recognized the prestige of theoretical physics among her earlier fellow students. She said that "many people that I knew they were like "oh yeah, theoretical physics" but that later on "most of them, they just went for other stuff like applied things because they also want to earn some money." This reflects an ideal physics practice as theoretical and pure.

The ranking of physics and other subjects and specializations within physics was described as a silly or immature idea by some of the students. Jan said that he did not respect other subjects before and that he and his friends were joking about both "chemists" and "applied 
physics" as undergraduates. When reflecting back on this, Jan pronounced these views as "adolescent," as views he does not hold anymore. One way of interpreting this is that the need for legitimizing one's own position by degrading others is stronger for novice students (Mendick et al. 2009). In a similar way, Bo, who had himself ranked subjects on a scale of coolness, claimed that "it's a bad habit for physicists" to "look down on other scientists in engineering." Bo thus draws from discourses of clear rankings of academic subjects and simultaneously resists these by drawing from discourses about equality and respect. While refuting and negotiating the discourses of ranking and belittlement, Jan and Bo at the same time confirm the prevalence of the idea that physics is better, and theoretical, pure physics is best.

Even though ranking sciences was described as immature by some of the students, the idea of a hierarchy of sciences is common, not only among students. Physics is often described as the most pure science and a model for others (Harding 1986). In research about the landscape of academic disciplines, it has been pointed out that a "placing of both knowledge fields and those that profess them in a finely tuned order of merit" is part of a general ethos of assessing value in academia (Becher and Trowler 2001, p. 191). The students can thus be understood as drawing from common discourses at universities that posit some subjects as superior to others. In this particular discourse, physics is seen as superior to other sciences and more pure and theoretical physics as especially celebrated.

\section{Negotiating Positions in Paths into Physics}

In analyzing the accounts of the interviewees in light of implicit rankings of subjects, patterns of negotiations in relation to study choice could be characterized. Several students described how a change of path made them re-evaluate their own place in a hierarchy of physicists. This was particularly important for those who moved down the supposed status ladder. In contrast, others who had "moved up" could feel confident about their choices. Furthermore, the few theoretical physics students did not seem to have ever experienced a need to think much about their place in the hierarchy of physics.

The perceived distinctions between different sub-fields of physics play an important role in interviewees' work for being recognized and recognizing themselves as competent physicists. However, some of the students were very confident in their choice of experimental or applied physics, even though they might recognize that their choice did not have the highest status among master's students. For example, Alex specifically emphasized his love of "tinkering" with machines, a (gendered) position of asserting competence as a "physically skilled physicist" identified in other studies (Danielsson 2009). Similarly, Eugenia asserted confidence in her aim to research materials for developing solutions to the world's energy problems. For her, identifying with experimental physics seemed straightforward, but was done in relation to an external goal, whereas Alex rather invested in a position as a machine-loving tinkerer. Another similar story was given by Elias, who initially did not have very specific ideas about where physics would take him. He had chosen physics instead of mathematics, which he liked more, because he thought it would give him "more options in the end." At the time of the interview, Elias expressed that he had developed an interest for medical physics since then, and could imagine doing research as well as working in other settings. These students do not seem to need to defend their choice of experimental or applied physics, perhaps because they do not see it as a step down from "higher" ambitions. In contrast to this, some students who have changed their direction did more work to negotiate their position and status as physicists. 
In the negotiations about status and imaginary physicist positions, the importance of doing or not doing theoretical physics was particularly important for two of the students, Sara and Valter. Here, the introductory quantum mechanics course, which can be seen as a gateway to theoretical physics, played a particular role as a turning point (see the in-depth discussion of this topic in Johansson 2018). In general, Sara's story is an example of a perceived downwards movement in the hierarchy of physics. Sara said that she was initially aiming at doing theoretical physics, and that she was very interested in both quantum mechanics and relativity already in high school. However, her physics studies did not proceed very smoothly and she had trouble when taking both these classes. A particularly significant event was an oral exam in relativity:

At first, I must say, I wanted to be like a theoretical physicist [...] But then I had a course, a relativity course with one of the professors that I admired a lot and I took a relativity class with him and then at the end of the course he told me 'now you can come and give an interview to improve your grade' and I didn't want to do it because I felt insecure about the things that I knew. But I went and then the interview was awful. He told me like 'are you sure that you want to be a physicist' and that really made me feel very bad. And he told me 'yeah, and you like theoretical physics, maybe you should look for something else'. So yeah (laugh), it made me feel very bad and I listened to him and I went to look for other stuff.

After this, Sara did her bachelor's project in an interdisciplinary setting, which was "not like physics, strict physics," but which she described had a very welcoming atmosphere, in contrast to what she had experienced among theoretical physics people. In describing her studies, Sara repeatedly referred to how among her previous and current peer students there were always some "people that understood everything because they are so smart," and how she was never one of them: "I know that I'm not a genius." In making sense of herself, Sara interprets her trajectory towards an interdisciplinary setting as a kind of failure, where a really successful physics student should be smart, "a genius," and ideally do theoretical physics. These statements posit Sara as a second-order physics student, who is not smart enough to go into the most celebrated subjects. This interpretation also largely puts the blame on the individual student, who is seen as the sole cause of failure (Johannsen et al. 2013), an attribution that is more common for women in STEM (LaCosse et al. 2016).

A similar story was told by Valter, who planned to study quantum informatics, but in the end found meteorology a subject which seemed more meaningful and concrete to him. Valter negotiated his trajectory between these fields in relation to the titles "meteorologist" and "physicist," and distanced himself from what people would normally associate with the title meteorologist: "the guy in the TV that points at the clouds and says "tomorrow you should wear a wind jacket." "He recognized the status difference in these titles, where physics is more "impressive" than meteorology, and said that he sometimes avoids the term "meteorologist" to avoid giving the wrong impression to people:

I think that if I say that I study physics or I study meteorology people will be more impressed by me if I say that I study physics. Despite maybe it is only my general bachelor instead of my precise field of study. [...] So I usually say that I study atmospheric physics, so I can hide behind this thing (laugh).

Valter described his change of path as leading to something that he found both more interesting and achievable. He related very explicitly to a discourse of ranking of physics subjects, and he 
evaluated his own position in relation to this. However, he placed these norms as external to himself, and posited himself as competent and interested in the subject he has chosen. In comparison to Sara, Valter did not construct his choices as clearly as a failure to do the more "impressive" physics of quantum mechanics or relativity, which he still described that he would like to learn more about at some time. Still, he clearly places himself in relation to a perceived hierarchy of physics and explicitly negotiates what the choice of something less "impressive" means to him.

Perceived status and competence can be negotiated in different ways, and being interdisciplinary can also be constructed as a successful path. One example of this is Bo, who switched to physics from materials engineering. In his evaluation this was an improvement, in that he considered physics to be cooler and less boring, and would find more pride in having a physics degree. At the time of the interview, Bo said that he still aimed to do research in materials, perhaps becoming an "electronics expert" but recognized that "to do some frontier research in these areas you need to be at least a half physicist." His broad education background was considered an asset in this. So, while he said that he at times envied his peer students who have a more solid background in physics and seemed to understand the current courses better, he posed his multidisciplinary path as a way to a specific and good career. This can be contrasted to the somewhat more negative sentiment of doing interdisciplinary work that is not "strict physics," put forward by Sara.

Neither of the two students who aimed for theoretical physics seemed to have needed to think much about their choices or their legitimacy in physics. Ivo described his choice of specialization as focused on what he was interested in, and he had not experienced any big problems in this. He had for example taken an advanced quantum field theory course to complete his bachelor's thesis, and although this was above his head in some sense, he now felt ready to complete his knowledge by learning the theory he needed. Jan, although discussing some of the troubles he had had with his own discipline for studying, never expressed any doubt about his choice or abilities. The kinds of negotiations visible in the stories of the other students are absent here. Issues of status and legitimacy are not as apparent to Ivo and Jan as to the other students. I interpret this as a consequence of the implicit affirmation they get in their position. They are recognized and recognize themselves as doing an appropriate and undeniably valued kind of physics and therefore do not need to negotiate their position as physicists as much as others.

\section{Resisting Celebrated Positions}

As detailed above, choosing physics means in some sense choosing to navigate among stereotypes about intelligence and nerdiness. This was needed already when the interviewees were in high school, but as has been described here, the master's students navigated similar discourses throughout their university studies in physics. However, the status of the nerdy genius doing theoretical physics is not indisputable, and some of the interviewees resist the status of this imaginary position in their narratives.

For Sara, some of the tension concerning intelligence in physics was played out in the group dynamics of her undergraduate studies. She explained that among her fellow bachelor's students, there was a "group of geniuses" who "didn't share anything" and "were always like 'oh yeah, I understand everything." 'Sara identified this group, consisting of a few students, as "the people that were more into theoretical physics." In particular, she recalled a fellow student 
who was "a part of us" from the start but did a bachelor's thesis in theoretical physics with the professor who had turned Sara down:

Sara: Now he's awful. He thinks that he knows everything and yeah, he's like becoming one of those guys that just think they know everything and feel so good about that.

I: Better than others in some way?

Sara: Yeah exactly, they are always like, talking about physics and if you don't know anything or you don't know something that they are mentioning, you're stupid, yeah.

Sara described this behavior as "they go Albert," indicating that these students invoke the powerful picture of Albert Einstein as a kind of ideal physicist which they strive to be like. For Sara, these students appear as authentically intelligent, but also as putting on a kind of show where this supposed intelligence may give status in physics. This status is achieved by appearing to possess the raw, authentic intelligence generally valued in western society and in that way being "effortless achievers," a position generally more available to privileged men (Castles 2012; Jackson and Nyström 2015; Paule 2015). However, Sara's identification of this behavior as a kind of act serves to make visible the positioning work that enables someone to appear as intelligent. In this way, I interpret Sara's distancing from obvious displays of intelligence as a possible act of resistance to intelligence norms.

While theoretical physicists may be viewed as smarter or better, those specializing in this subject area can also be seen as more nerdy. Bo, when discussing how physics students can generally be viewed as crazy in focusing on very difficult topics pointed out that "Actually, in my view I think students in theoretical physics are more crazy than me (laugh)." I interpret this picture of the more "crazy" theoretical physicist as part of the nerd complex of attributes surrounding the physicist stereotype. Dan, who studies geophysics, compared his classmates with those supposedly more nerdy students studying quantum physics in their master's:

I mean we are a select group of geophysicists and we get along quite well already I think $[\ldots]$ yeah and I think it's a nice group yeah ... more or less like-minded in a way well, not too nerdy (laugh) if you know what I mean, because in quantum physics you probably have some ... I have no idea but ... I can imagine something like that (laugh), so I think the people [in geophysics] are pretty cool.

Being nerdy is constructed as undesirable compared to being one of "the cool people you would meet on geophysics," as Dan says, "the more normal people who go to parties and have a life outside physics." In this discussion, the word cool takes on a different meaning to how Bo used it to characterize more or less interesting subjects. Here, cool refers to being laid-back and everyday. It emphasizes the "ordinariness" of the self in relation to an imaginary position recognized as over-the-top in some aspects (Wetherell and Edley 1999). Dan's statements can be seen as a defense against a perceived norm posing geophysics students as less intelligent than other physics students. At the same time Dan admits that "I don't think there's like a genius among the geophysicists," but that those people would rather "most likely go to theoretical physics." However, in Dan's account, this figure of the "genius" physics student is so clearly associated with being "socially awkward" that it cannot be constructed as a really desirable position, invoking the abject, disdained position of the nerd (Francis 2009; Willey and Subramaniam 2017). Here, the general discourses about nerdiness reappear and are rearticulated in the context of physics master's education.

In summary, most of the interviewees recognize an imaginary position of the intelligent, theoretical physics student as something they have to relate to. While some do not need to 
negotiate this position, others construct complementary valuable positions as good experimentalists, interdisciplinary specialists, or socially engaged scientists. Still others may more explicitly resist the position of the intelligent but perhaps nerdy physics student who "goes Albert," for example by investing in a position as a cool and ordinary physics person.

\section{Discussion}

This article set out with the aim of exploring the negotiations of positions needed for physics master's students attempting to be recognized as competent future physicists. In particular, discourses about intelligence and nerdiness circulating inside and outside physics, and the implications of the choice of physics subjects have been shown to play an important role in the negotiations of physics students. The stereotype of physicists as intelligent and nerdy has been well-described in the literature and the rankings of physics subjects described here may not come as a surprise to a physicist. However, how these discourses play out in the negotiations of master's students has not been described before. This article has shown that the ideal of intelligent, absorbed geniuses serve as a powerful imaginary position against which physics students must negotiate their own place in physics. While it can be argued that a certain kind of intelligence and interest is required for physics, the results show that the consequences of a strong idealization of these characteristics can carry significant costs to students and physics education, as expectations of intelligence, passion, and nerdiness all can serve to limit the possibilities for identifying as a physicist.

The study presented in this article is based on interviews with a limited number of physics students, which means that the results must be interpreted as cases to learn from rather than generalizable data (Flyvbjerg 2006). A more comprehensive or longitudinal research design may lead to different results. Nevertheless, the international reach of the sample indicates that students in different contexts of physics education encounter similar discourses about intelligence, nerdiness, and authenticity when it comes to becoming a physicist. The focus of the interviews has been to put the interviewees' stories and experiences first, and this may have lead to a less comprehensive account of their pathways into physics than a more strict chronological interview guide could have given. For example, only a few students described important key episodes during their undergraduate studies. However, more detailed accounts of what the students' currently experienced as significant could be achieved in this way. The results of this study are based on a qualitative interpretation of the interviewees' statements, which means that there is room for different interpretations. The comprehensive analysis however establishes the results as viable interpretations of the current material. In light of this, the results should be viewed as another piece in our tentative understanding about how common discourses in physics and science education can affect students' negotiations of physicist positions.

As indicated by the stories of several of the students in this study, intelligence has significant currency in physics. Whether you are smart enough or not becomes a primary indicator of your physics credibility. The issue here is not necessarily to do with intelligence or cognitive capacity per se, but with the social recognition of intelligence. In physics, the ideal, or highest stage of a physics career, is a kind of genius, the smart super-physicist (Traweek 1988). As the embodiment of this ideal is generally seen as male, achieving recognition in other, less celebrated positions may be necessary for women (Gonsalves and Seiler 2012). This article has demonstrated how this social recognition of intelligence comes to matter for 
master's students. One expression of this can be seen in the investment in "brainy" positions by some physics students, something which, with a phrase borrowed from Sara, can be called "going Albert." Regardless of the competence and capabilities of these students, the possibilities for taking such a position are structured by gendered and classed notions of who can be a genius (Jackson and Nyström 2015; Paule 2015).

Physics is a special choice, not something that every teenager is expected to strive for, and physics students are usually driven by curiosity and interest (Levrini et al. 2017), if not a downright passion for understanding the universe. We have seen how being recognized as an interested or even nerdy student may be a way of finding a position removed from for example expectations of getting a "good job," evidenced by the feeling of belonging when coming to physics reported by some of the interviewees. Here, both gender and social background are important ingredients in feeling at home in physics. The interest expected of a physics student can however also be experienced as a demand for being an authentic physics person, of always having had this intense interest, as Susanna's story indicates. In this way, both the expected intelligence and absorption of physics students can serve to exclude people who are not as readily expected to harbor these qualities: women, minorities, working class, etc. (Mendick et al. 2009).

The captivation expected of physicists is always bordering on nerdiness. However, as described in this article and in previous research, the "nerd" label often carries negative connotations. At the same time, being "nerdy enough" can serve as an in-group qualification, for example by getting the jokes (Hasse 2008). There are, however, also norms about what kind of passion, what type of nerdiness, is counted as most celebrated or appropriate for a physicist. Being passionate about understanding the big questions about the universe, solving deep mysteries, etc., corresponds to the stereotype of the genius super-physicist. Being passionate about producing new materials to solve the world's energy problems, like Eugenia, just does not meet that criterion, and is indeed a driving force for very few physics students (Levrini et al. 2017). A society-oriented motivation for studying science is in general more common for women, but less common for physics students (Eccles 2007; Sax et al. 2016; Schreiner 2006) and does not correspond to the ideal of the male genius-nerd who focuses on "pure" physics as the most revered kind of physics.

In the discourses recognized by the students interviewed for this study, theoretical specializations of physics are considered harder and as requiring more intelligent students. While several students acknowledge condescending attitudes in line with these beliefs as inappropriate and immature, something an experienced student should have left behind, the implicit ranking still plays an important role in making sense of one's position in physics. One of the consequences of this thinking is that some students, by virtue of their subject choice, are considered smarter than others, and that any move away from the higher status, theoretical subjects, can be interpreted as proof that you were not smart enough. As we have seen, one defense against being viewed as less smart due to choosing an experimental, applied, or less fundamental subject is by investing in a position as a non-nerdy physicist, which rejects the status of more pure and theoretical physics due to its supposed nerdiness. However, with intelligence being a central currency in physics and some fields by default being viewed as demanding more or less intelligence, the available positions as a legitimate physics student become constrained. In addition, norms about authentic intelligence and how it is exhibited affect students' self-efficacy and study choice. Attributing failures to one's own shortcomings has been shown to be more common for women in STEM (LaCosse et al. 2016), and if genius status and authentic intelligence are more easily attributed to men, the problem with "fixed 
mindsets" about intelligence (Dweck 2006) becomes compounded with gender. This suggests that the notion that one is not cut out for the supposedly more intelligent and higher status theoretical fields of physics is more easily ascribed to and accepted by female physics students.

\section{Implications}

This article deepens the picture of physics education at graduate level by focusing on sociocultural questions of identity, a topic which has been underdeveloped in physics education research where the primary topic of interest has been the cognitive aspects of learning. Among other things, the results show how physics education cannot be considered a similar experience for all students and how divisions among physics subjects are important for students' social experiences of studying physics. Extending the research of science and physics identities in school and undergraduate university studies, the results show how the common stereotypes of physicists as intelligent and nerdy continue to play an important role for how students at the master's level make sense of themselves and their position in physics. This shows how, even though the students have already passed through the initial stages as newcomers in physics and received their first degree, the possibilities for being recognized as successful are dependent on factors such as subject specialization, gender, and social background. These results contribute to a more detailed picture of the sociocultural aspects of physics studies, adding to the body of knowledge about study choice, persistence, and equity in physics.

In future studies, it would be worthwhile to extend these lines of inquiry in several ways. Investigating the negotiations of students in other subjects that are considered prestigious in one way or another might provide interesting points of comparison to the results reported here. Tacit status evaluations among and between academic disciplines are common (Becher and Trowler 2001), and how students relate to the ideals of their respective field, be it in STEM or outside, is an important topic to explore. This study represents a snapshot of student experiences with a limited cohort of physics students, and the interviewees' often refer to earlier experiences and attitudes. In light of this, it would be informative to extend and contrast the results reported here by asking similar questions with a more longitudinal research design. Such research could ask what different positions students negotiate during their studies and whether/why the situation changes over time.

Ideas of authentic intelligence have been shown to be a possible stumbling block for learning, as a "fixed mindset" about intelligence implies expending effort is fruitless (Dweck 2006). Then again, the position of an effortless achiever or a genius is already gendered and classed (Jackson and Nyström 2015; Paule 2015). The results of this article point to how ideals of authentic intelligence, effortless achievement, and nerdy interest are reproduced and negotiated among master's level physics students. I do not intend to imply that there is no need for physics students to be intelligent, interested, and engaged to some extent, but rather that these normative ideals may make many ways of being a physicist less imaginable as well as inhibit learning. The question is not about who can manage physics studies (which all of the interviewees have done), but a question of how normative discourses promote specific ways of being a physicist over others. These discourses give affirmation to some (predominantly white, male) students, who can get recognition as some sort of "geniuses." However, this can eventually inhibit learning for all students, since putting in effort is seen as futile. The implicit ranking of the subfields of physics and their practitioners is something that is reflected as students make sense of their positions and something that needs to be considered when 
teaching physics. What signals do physics instructors communicate to students? Do we wish to accept a picture positing some choices as mainly being for those who cannot cut it for the difficult, higher status fields? Considering and counteracting possibly harmful discourses in physics education is an important part of making physics more diverse and inclusive.

\section{Conclusions}

This study adds to the literature on social identity in science education by highlighting how discourses about intelligence and nerdiness that are relatively well-documented in high-school and undergraduate physics play a role also at the master's level of physics studies. In the analyzed interview material, these discourses were found to impact the evaluation of physics subjects as requiring more or less intelligence and therefore being accorded higher or lower status. The interviewees related to a revered imaginary position of the brilliant genius physicist doing theoretical work and the negotiations around this ideal concern both fitting in as a physicist and fitting in as a certain type of physicist. The interviewed students have negotiated these common discourses in different ways, and the results indicate that much identity work was required in particular for those students who had to re-evaluate their study choice from theoretical to other physics, thus crafting positions as "another type of physicist" than their initial ideal. In addition, women and students from less study-oriented environments are disfavored by the expectations of who the typical, authentic physics student should be, and in this study consequently had to do more work for negotiating a position in physics. Resistance to these discourses could be seen in the negotiations over nerdiness or arrogance, where positions as non-nerdy or sympathetic physicists were seen as viable alternatives to the revered position. In the end, the deeper knowledge of the normative discourses affecting physics students' experiences and study choices detailed in this study can be used to create a more inclusive environment in physics programs around the world.

Acknowledgments I would like to thank all those who have read, discussed, and provided valuable feedback during this project. In particular, Minna Salminen-Karlsson and Maja Elmgren deserve a heartfelt thanks for all you have contributed to my thinking and writing on the topic. I am also grateful for the help given by John Airey, Staffan Andersson, Klara Goedecke, Johanna Larsson, and the Education and Science Group at the Centre for Gender Research.

Open Access This article is distributed under the terms of the Creative Commons Attribution 4.0 International License (http://creativecommons.org/licenses/by/4.0/), which permits unrestricted use, distribution, and reproduction in any medium, provided you give appropriate credit to the original author(s) and the source, provide a link to the Creative Commons license, and indicate if changes were made.

\section{References}

Archer, L., Dawson, E., DeWitt, J., Godec, S., King, H., Mau, A., Nomikou, E., \& Seakins, A. (2017). Killing curiosity? An analysis of celebrated identity performances among teachers and students in nine London secondary science classrooms. Science Education, 101(5), 741-764 10/gctkbf.

Archer, L., Moote, J., Francis, B., DeWitt, J., \& Yeomans, L. (2016). The "exceptional" physics girl: a sociological analysis of multimethod data from young women aged 10-16 to explore gendered patterns of post-16 participation. American Educational Research Journal, 1-39 10/gctkdj.

Becher, T., \& Trowler, P. (2001). Academic tribes and territories: intellectual enquiry and the culture of disciplines (2nd ed.). Philadelphia: Open University Press.

Bøe, M. V., \& Henriksen, E. K. (2013). Love it or leave it: Norwegian students' motivations and expectations for post-compulsory physics. Science Education, 97(4), 550-573 10/f42pp9. 
Butler, J. (1990). Gender trouble: feminism and the subversion of identity. New York: Routledge.

Carlone, H. B., \& Johnson, A. (2007). Understanding the science experiences of successful women of color: Science identity as an analytic lens. Journal of Research in Science Teaching, 44(8), 1187-1218 10/cpprr8.

Carlone, H. B., Scott, C. M., \& Lowder, C. (2014). Becoming (less) scientific: a longitudinal study of students' identity work from elementary to middle school science. Journal of Research in Science Teaching, 51(7), 836-869 10/f6dgcr.

Castles, E. E. (2012). Inventing intelligence: how America came to worship IQ. Santa Barbara, Calif: Praeger.

Connell, R. W. (1995). Masculinities. Cambridge: Polity Press.

Danielsson, A. T. (2009). Doing physics — doing gender: an exploration of physics students' identity constitution in the context of laboratory work. PhD thesis, Uppsala University http://urn.kb.se/resolve?urn= urn:nbn:se:uu:diva-98907.

DeWitt, J., Archer, L., \& Osborne, J. (2013). Nerdy, brainy and normal: children's and parents' constructions of those who are highly engaged with science. Research in Science Education, 43(4), 1455-1476 10/f4465k.

Dweck, C. S. (2006). Mindset: The new psychology of success. New York: Random House http://catdir.loc. gov/catdir/enhancements/fy0624/2005046454-b.html. Accessed 15 December 2017.

Eccles, J. S. (2007). Where are all the women? Gender differences in participation in physical science and engineering. In S. J. Ceci \& W. M. Williams (Eds.), Why aren't more women in science?: top researchers debate the evidence. (pp. 199-210). Washington, DC: American Psychological Association. doi:10/cp6w4z.

Eglash, R. (2002). Race, sex, and nerds: from Black geeks to Asian American hipsters. Social Text, 20(2), 49-64.

Flyvbjerg, B. (2006). Five misunderstandings about case-study research. Qualitative Inquiry, 12(2), 219-245 10/fsd688.

Francis, B. (2009). The role of The Boffin as abject Other in gendered performances of school achievement. The Sociological Review, 57(4), 645-669 10/d23p2x.

Francis, B., Archer, L., Hodgen, J., Pepper, D., Taylor, B., \& Travers, M.-C. (2017a). Exploring the relative lack of impact of research on 'ability grouping' in England: a discourse analytic account. Cambridge Journal of Education, 47(1), 1-17 10/gctkbr.

Francis, B., Archer, L., Moote, J., DeWitt, J., MacLeod, E., \& Yeomans, L. (2017b). The construction of physics as a quintessentially masculine subject: young people's perceptions of gender issues in access to physics. Sex Roles, 76(3-4), 156-174 10/f9nnjt.

Gonsalves, A. J. (2014). "Physics and the girly girl-there is a contradiction somewhere": doctoral students' positioning around discourses of gender and competence in physics. Cultural Studies of Science Education, 9(2), 503-521 10/gctkz2.

Gonsalves, A. J., Danielsson, A., \& Pettersson, H. (2016). Masculinities and experimental practices in physics: the view from three case studies. Physical Review Physics Education Research, 12(2), 020120 10/f3rnhx.

Gonsalves, A. J., \& Seiler, G. (2012). Recognizing "smart super-physicists": gendering competence in doctoral physics. In M. Varelas (Ed.), Identity construction and science education research (pp. 157-172). Rotterdam: SensePublishers 10/cs4t.

Hall, S. (1996). Who needs ‘identity’? In S. Hall \& P. Du Gay (Eds.), Questions of cultural identity (pp. 1-17). London: Sage.

Harding, S. (1986). The science question in feminism. Ithaca: Cornell University Press.

Hasse, C. (2008). Learning and transition in a culture of playful physicists. European Journal of Psychology of Education, 23(2), 149-164 10/cxg4zs.

Hasse, C. (2015). The material co-construction of hard science fiction and physics. Cultural Studies of Science Education, 10(4), 921-940 10/gctks3.

Hasse, C., \& Sinding, A. B. (2012). The cultural context of science education. In D. Jorde \& J. Dillon (Eds.), Science education research and practice in Europe (pp. 237-252). Rotterdam: SensePublishers 10/cs4v.

Hasse, C., Trentemøller, S., \& Bjerregaard Sinding, A. (2008). Draw the line!: international conference, Copenhagen 2008: papers, proceedings and recommendations. Copenhagen: UPGEM, Understanding Puzzles in the Gendered European Map. https://cordis.europa.eu/publication/rcn/10454_en.html

Hazari, Z., Potvin, G., Lock, R. M., Lung, F., Sonnert, G., \& Sadler, P. M. (2013). Factors that affect the physical science career interest of female students: testing five common hypotheses. Physical Review Special Topics-Physics Education Research, 9(2), 020115 10/gctkvg.

Hazari, Z., Sonnert, G., Sadler, P. M., \& Shanahan, M.-C. (2010). Connecting high school physics experiences, outcome expectations, physics identity, and physics career choice: a gender study. Journal of Research in Science Teaching, 47(8), 978-1003 10/fwt79h.

Holmegaard, H. T., Ulriksen, L., \& Madsen, L. M. (2015). A narrative approach to understand students' identities and choices. In E. K. Henriksen, J. Dillon, \& J. Ryder (Eds.), Understanding student participation and choice in science and technology education (pp. 31-42). Dordrecht: Springer 10/cs4s.

Jackson, C., \& Nyström, A.-S. (2015). 'Smart students get perfect scores in tests without studying much': why is an effortless achiever identity attractive, and for whom is it possible? Research Papers in Education, 30(4), 393-410 10/f3mv67. 
Johannsen, B. F., Rump, C. Ø., \& Linder, C. (2013). Penetrating a wall of introspection: a critical attrition analysis. Cultural Studies of Science Education, 8(1), 87-115 10/f22r2w.

Johansson, A. (2016). Analyzing discourse and identity in physics education: Methodological considerations. In D. L. Jones, L. Ding, \& A. L. Traxler (Eds.), 2016 Physics Education Research Conference Proceedings (pp. 180-183). https://doi.org/10.1119/perc.2016.pr.040.

Johansson, A. (2018). Undergraduate quantum mechanics: Lost opportunities for engaging motivated students? European Journal of Physics, 39(2), 025705. https://doi.org/10.1088/1361-6404/aa9b42.

Keller, E. F. (1985). Reflections on gender and science. New Haven, Conn.: Yale University Press.

Kendall, L. (2000). “OH NO! I'M A NERD!": hegemonic masculinity on an online forum. Gender \& Society, 14(2), 256-274 10/dzch7z.

Kerr, B. A., \& Kronborg, L. (2015). Editorial. Gifted and Talented International, 30(1-2), 1-5 10/gctj9j.

Laclau, E., \& Mouffe, C. (1985). Hegemony \& socialist strategy. London: Verso.

LaCosse, J., Sekaquaptewa, D., \& Bennett, J. (2016). STEM stereotypic attribution bias among women in an unwelcoming science setting. Psychology of Women Quarterly, 40(3), 378-397 10/gcwckk.

Leslie, S.-J., Cimpian, A., Meyer, M., \& Freeland, E. (2015). Expectations of brilliance underlie gender distributions across academic disciplines. Science, 347(6219), 262-265 10/f6ttq3.

Levrini, O., Ambrosis, A. D., Hemmer, S., Laherto, A., Malgieri, M., Pantano, O., \& Tasquier, G. (2017). Understanding first-year students' curiosity and interest about physics-lessons learned from the HOPE project. European Journal of Physics, 38(2), 025701 10/gctkb9.

Macdonald, A. (2014). "Not for people like me?": under-represented groups in science, technology and engineering. Bradford, UK: WISE. http:/www.wisecampaign.org.uk/education/not-for-people-like-me

Madsen, L. M., Holmegaard, H. T., \& Ulriksen, L. (2015). Being a woman in a man's place or being a man in a woman's place: Insights into students' experiences of science and engineering at university. In E. K. Henriksen, J. Dillon, \& J. Ryder (Eds.), Understanding student participation and choice in science and technology education (pp. 315-330). Dordrecht: Springer 10/cs4r.

Maltese, A. V., \& Tai, R. H. (2010). Eyeballs in the fridge: sources of early interest in science. International Journal of Science Education, 32(5), 669-685 10/fbrk7x.

Mendick, H., \& Francis, B. (2012). Boffin and geek identities: abject or privileged? Gender and Education, 24(1), 15-24 10/d4cvzm.

Mendick, H., Moreau, M.-P., \& Epstein, D. (2009). Special cases: Neoliberalism, choice and mathematics education. In L. Black, H. Mendick, \& Y. Solomon (Eds.), Mathematical relationships: identities and participation (pp. 71-82). Abingdon: Routledge.

Merchant, C. (1989). The death of nature: women, ecology, and the scientific revolution. San Francisco: Harper \& Row.

Ong, M. (2005). Body projects of young women of color in physics: intersections of gender, race, and science. Social Problems, 52(4), 593-617 10/bpb6tz.

Paule, M. (2015). Dinosaur discourses: taking stock of gendered learning myths. Gender and Education, 27(7), $744-758$ 10/gctj9m.

Pozzer, L. L., \& Jackson, P. A. (2015). Conceptualizing identity in science education research: Theoretical and methodological issues. In C. Milne, K. Tobin, \& D. DeGennaro (Eds.), Sociocultural studies and implications for science education (pp. 213-230). Dordrecht: Springer 10/cs4w.

Privateer, P. M. (2006). Inventing intelligence: a social history of smart. Malden: Blackwell Pub.

Pullman, P. (1995-2000). His dark materials. Scholastic: Book series.

Sax, L. J., Lehman, K. J., Barthelemy, R. S., \& Lim, G. (2016). Women in physics: a comparison to science, technology, engineering, and math education over four decades. Physical Review Physics Education Research, 12(2), 020108 10/gctq4v.

Schreiner, C. (2006). Exploring a ROSE-garden: Norwegian youth's orientations towards science — seen as signs of late modern identities. PhD thesis, University of Oslo. http://urn.nb.no/URN:NBN:no-12326

Storage, D., Horne, Z., Cimpian, A., \& Leslie, S.-J. (2016). The frequency of "brilliant" and "genius" in teaching evaluations predicts the representation of women and African Americans across fields. PLOS ONE, (3), 11, e0150194 10/f8whhw.

Taylor, P. C. (2014). Contemporary qualitative research: toward an integral research perspective. In S. K. Abell \& N. G. Lederman (Eds.), Handbook of research on science education (Vol. II, pp. 38-54). New York: Routledge.

Traweek, S. (1988). Beamtimes and lifetimes: the world of high energy physicists. Cambridge, Mass.: Harvard University Press.

Traxler, A. L., Cid, X. C., Blue, J., \& Barthelemy, R. (2016). Enriching gender in physics education research: a binary past and a complex future. Physical Review Physics Education Research, 12(2), 020114 10/gctkdz.

Wajcman, J. (1991). Feminism confronts technology. Cambridge: Polity Press. 